Communication Systems Division (SSC)

EPFL CH-1015 Lausanne, Switzerland

http://sscwww.epfl.ch

\title{
Design Protection for WDM Optical Networks ${ }^{1}$
}

\author{
O. Crochat \\ J.-Y. Le Boudec \\ Laboratoire de Reseaux de Communication (LRC) \\ EPFL - DI \\ CH - 1015 Lausanne \\ Tel: +41216936601 \\ Fax: +41216936610 \\ crochat@lrc.epfl.ch \\ leboudec@di.epfl.ch
}

March 1997

\footnotetext{
${ }^{1}$ This work is supported in part by the ACTS project COBNET and the Swiss government (OFES) and represents the view of the authors
}

Technical Report SSC/1997/005 


\begin{abstract}
With WDM networks, the failure of a single link or component may cause the simultaneous failure of several optical channels, potentially making impossible the restoration by rerouting in higher layers directly using the optical network (SDH, ATM, IP). To address this, we introduce the concept of Design Protection, which aims at making such failure propagations impossible. We present the Disjoint Alternate Path (DAP) algorithm which places optical channels in order to maximise design protection. We show the result on the example of the ARPA-2 network.
\end{abstract}

Keywords: WDM, optical network, survivability, routing, tabu search. 


\section{Introduction}

The large bandwidth of optical fibres have made them attractive for high-speed networks. The use of Wavelength Division Multiplexing (WDM) allows aggregation of many channels onto a single fibre without the need of high-speed optoelectronic devices for end-users $[1,2,3,4,5]$. It is the simplicity of this multiplexing scheme, permitting a relatively easy access to the fibre's bandwidth, which is the underlying reason why WDM is about to play a major role in the expansion of photonic networks.

However the existence of many independent data channels over the fibre infrastructure could lead to problems in case of failure, as the amount of bandwidth lost by a component or cable failure is now much larger than what would have been lost in a traditional network. This is why extra effort must be spent in analyzing optical WDM networks and in finding ways to protect them against failures $[6,7,8]$.

\subsection{Definitions}

We consider a backbone WDM photonic network consisting of optical crossconnect nodes (or simply nodes) interconnected by optical bidirectional links (see bottom of Figure 1). Each link actually consists of a pair of unidirectional fibres and is capable of transporting a limited number of wavelength channels. We assume that a node is capable of routing any incoming wavelength channel on any of the incoming fibres, to any wavelength channel on any of the outgoing fibres. The node may be performing all cross-connecting functions purely in the optical domain (All-Optical Networks) $[9,10]$ or, as in the COBNET project [11], using a hybrid electrical-optical system (we do not make any distinction between these cases in this paper).

- A Network Port is a point on a node which gives access to the photonic network. Connected to a network port, one might have an SDH crossconnect, an ATM node, or even an IP router (called higher layer switches). In the last two cases, the ATM or IP layer uses a physical channel such as SDH or Fibre Channel to carry the cells or packets. A network port is composed of the optoelectronic devices required to connect one "lowspeed" end-user to the high-speed optical network. Each node contains a certain number of ports.

- An Optical Link is a bidirectionnal physical connection between two nodes.

- A Clear-Channel (CC) is a bidirectionnal communication channel between two ports (like $c 6$ between ports $B 2$ and $C 3$ in Figure 1). The Route of a clear-channel is the list of optical links used by the clear-channel (in Figure 1, the route of clear-channel $c 6$ has been - arbitrarily - chosen as $(l 4, l 5))$.

- A Path between two ports is a concatenation of clear-channels that are interconnected in the higher layer (SDH, ATM or IP). Figure 1 shows 
how paths, clear-channels and optical links are related. A path can be Single-hop, if only one clear-channel is used (path $p 1=(c 6)$ between $B$ and $C$ in Figure 1 ) or Multihop, if more than one is used (for example path $p 2=(c 4, c 5, c 3)$ between $B$ and $C)[12,13]$.

Note that, with these definitions, hops refer to higher layer switching; a single-hop path will usually traverse more than one optical cross-connect node. A multi-hop path is not a clear-channel, and is protocol-specific (as all the clear-channels used must conform to the same protocol).

The Virtual Topology consists of a graph representing all the clear-channels that are present in the network. It is the only view of the network available to the higher layer switches. The Physical Topology is the real network, composed of optical links and photonic nodes. The mapping between these two topologies (i.e. the assignment of the clear-channels within this network) is performed by the design algorithm.

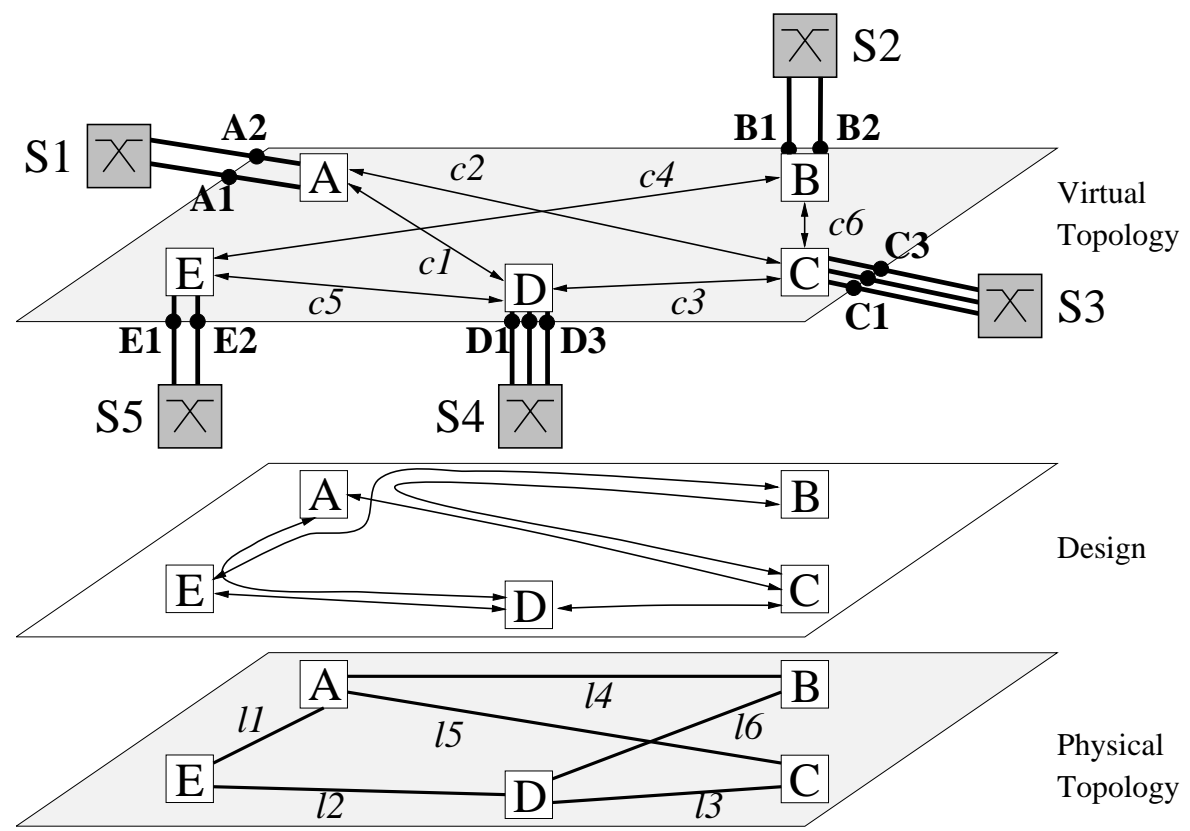

Figure 1: The two topologies and a design example. Clear-channel $c 6$ uses optical links $l 4$ and $l 5$. There is a single-hop path $p 1=(c 6)$ between $B$ and $C$, as well as two alternate, multi-hop paths $p 2=(c 4, c 5, c 3)$ and $p 3=(c 4, c 5, c 1, c 2)$ 


\subsection{Protection Mechanisms}

Physical Protection has the objective of ensuring that a bidirectional communication channel between two network ports will remain operational in the event of a component or an optical link failure. This protection is performed at the physical level. It can react very rapidly to such failure, but requires hardware redundancy $[8,14]$. In general, physical protection also includes the ability to re-route clear-channels after a failure, without the higher level switches being aware of it [6].

There is a price to pay for Physical Protection, namely that of optical hardware duplication. In many situations it may not be cost-effective to provide such redundancy, and thus in real networks, we may only find physical protection implemented on business-critical routes. For other routes in such networks, clear-channel failures will occur. This should normally not result in dramatic losses of service because the higher layers (for example IP routers) have their own protection mechanisms. However, this may not always be true. Consider again Figure 1. The virtual topology seen by the higher layer seems to offer adequate redundancy between the higher layer switches (or routers). Assuming that clear-channel $c 6$ fails, the higher layer will be able, thanks to its own routing algorithm, to reroute traffic between $B$ and $C$ through the alternate path $p 2=(c 4, c 5, c 3)$. In reality however, clear-channels $c 6$ and $c 4$ share a common optical link $l 4$. If $l 4$ fails there is a simultaneous failure of $c 4$ and $c 6$ in the virtual network.

The higher layers have a general view of the network where clear-channels are independent. Their own protection mechanisms (like rerouting algorithms or spare capacity allocation on the clear-channels) are based on this independence assumption. The previous example shows that this is not always true if WDM is used in the lowest layer. The purpose of Design Protection is to avoid such situations, by a proper placement of clear-channels onto optical links. In other words, the objective is to produce virtual networks for which the assumption of clear-channels independence is true. Of course it is still necessary for the higher layers to plan spare capacities inside the clear-channels in order to make service restoration possible.

Section 2 introduces the concept of Design Protection, and gives its mathematical formulation. Section 3 presents the Disjoint Alternate Path (DAP) algorithm, an algorithm using tabu search for placing clear-channels in order to maximize design protection while trying to enforce link capacity constraints. Section 4 shows the results obtained by the algorithm in various cases and discuss them.

\section{Design Protection}

The cost of physical protection and the guarantee of connectivity between network ports may be prohibitively high for its implementation in many situations. In this section, we consider a weaker form of network protection, called Design 
Protection, which aims at avoiding simultaneous failures in the virtual network.

\subsection{The Principle}

We have explained in Section 1.2 how hidden dependences between clear-channels could make it impossible for the higher layer to reroute around a failure, in spite of apparent redundancy in the virtual network. The aim of Design Protection is to find, from a given virtual topology, a route for each of the clear-channels in the photonic network such that a single optical link failure leaves the virtual network connected. For example, a placement of clear-channels that satisfies this requirement is shown in Figure 2.

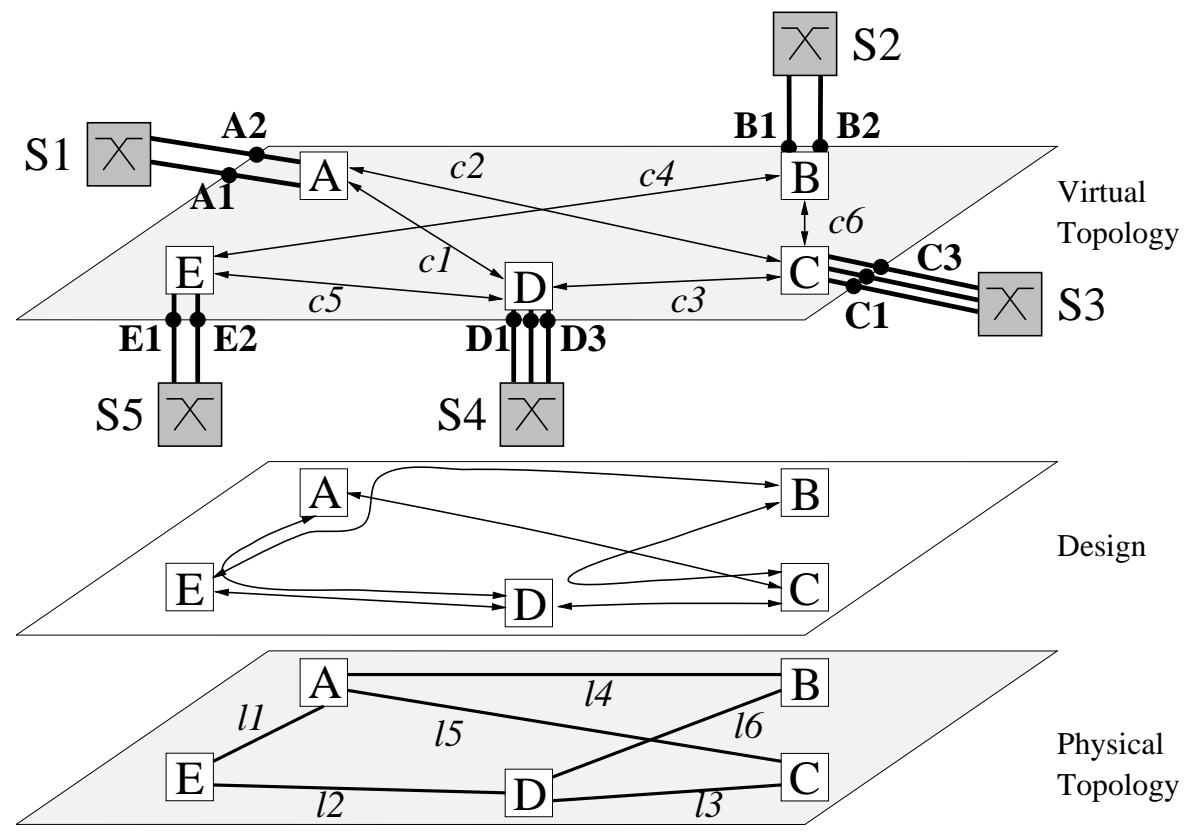

Figure 2: Same physical and virtual topologies as in Figure 1 but with a slightly different design (the route of $c 6$ is now $(l 6, l 3)$ ), allowing survivability to any one link failure in the physical topology

Design Protection is performed by the configuration management at setup time, through the design algorithm which maps the clear-channels described by the virtual topology onto the physical topology. It should be noted that this design protection is a static protection i.e. we are not considering a dynamic reconfiguration of the photonic nodes in the network following a node or link failure.

Design protection uses the indicated physical and virtual topologies. The physical topology may be computed by an algorithm as a function of installa- 
tion costs, inter-node distances or other parameters, or may be fixed due to an already installed fibre infrastructure [15]. The virtual topology may be computed by an algorithm trying to optimize distances, delays or number of hops between higher-layer routers connected to this network $[16,9,10]$.

One should notice that this discussion about resilience against optical link failures can easily be adapted to node failures. The difference is that the routes of a clear-channel and its associated alternate path must be node-disjoint (instead of link-disjoint for link failure protection). However, in the case of node failures, it is not possible to protect all the clear-channels that had a port in the broken node, as all the end-users connected to this node are now unreachable. The only achievable protection is against failure propagation for the remaining clear-channels.

\subsection{Formulation of the problem}

We develop in this section a general model and formulate the design problem as an optimization problem. The physical topology is represented by an undirected graph, $G_{p}$, where each node corresponds to a node in the network, and each edge to an optical link. The virtual topology is represented by an undirected graph, $G_{v}$, where each node corresponds to a node in the network, and each edge to a clear-channel.

The following notation is used:

- $s, d$ are used to denote end-points of clear-channels

- $m, n$ are used to denote end-points of optical links

\section{Given}

- Let $N$ be the number of nodes in the network.

- Let $L=\left(l_{m n}\right)$ be the $N \times N$ matrix representing the physical topology, where

$l_{m n}\left(=l_{n m}\right)$ is the number of wavelength channels available on this link.

Note that $l_{m n}=0$ signifies that there is no optical link between $m$ and $n$

- Let $C=\left(c_{s d}\right)$ be the $N \times N$ matrix representing the virtual topology, i.e.,

$$
c_{s d}=c_{d s}= \begin{cases}1 & \text { if there is a clear-channel between } s \text { and } d \\ 0 & \text { otherwise }\end{cases}
$$

Primary variable A solution to the problem consists of a set of values for all the $\mathcal{R}_{s d}$ matrices. 
- Clear-Channel Design:

The $N \times N$ matrix $\mathcal{R}_{s d}=\left(r_{m n}^{s d}\right)$ represents the route used by the clearchannel $c_{s d}$ on the physical topology, where

$$
r_{m n}^{s d}=r_{n m}^{s d}= \begin{cases}1 & \text { if } c_{s d} \text { uses link } l_{m n} \text { on its route } \\ 0 & \text { otherwise }\end{cases}
$$

Auxiliary variables Those variables are obtained from the primary variable and the given constants. They help in formulating the optimization criteria.

- $\bar{C}_{m n}=\left(\bar{c}_{s d}\right)$ is the $N \times N$ matrix representing the virtual topology after having removed all the clear-channels $c_{s d}$ that have $l_{m n}$ or $l_{n m}$ as part of their route $\mathcal{R}_{s d}$

$$
\bar{c}_{s d}= \begin{cases}0 & \text { if }\left(c_{s d}=0\right) \cup\left(r_{m n}^{s d}=1\right) \cup\left(r_{n m}^{s d}=1\right) \\ 1 & \text { otherwise }\end{cases}
$$

- $b_{m n}^{s d}$ equals 1 if clear-channel $c_{s d}$ has no alternate path when link $l_{m n}$ is broken,

$$
b_{m n}^{s d}= \begin{cases}1 & \text { if }\left(c_{s d}=1\right) \cap\left(l_{m n}>0\right) \cap\left(s \text { and } d \text { unconnected in } \bar{C}_{m n}\right) \\ 0 & \text { otherwise }\end{cases}
$$

- A broken pair is a couple $\left\{c_{s d}, l_{m n}\right\}$ for which $b_{m n}^{s d}=1$.

\section{Constraints}

- Clear-channels use existing links and do not exceed the overall capacity of each link:

$$
\sum_{s, d} r_{m n}^{s d} \leq l_{m n} \quad \forall m, n
$$

- $\mathcal{R}_{s d}$ represents a sequence of nodes where no node is repeated.

$$
\sum_{m} r_{m n}^{s d}= \begin{cases}1 & \text { if } m=s \text { or } d \\ 2 & \text { if node } n \text { is on the route of } c_{s d} \\ 0 & \text { otherwise }\end{cases}
$$

Note that there is no constraint on the number of ports (transmitter, receiver) per network node. This is a constraint for the virtual topology design algorithm $[9,10]$, and we assume that the given virtual topology of Equation 2 fulfill these constraints. 
Optimality Criterion The aim is to minimize the number of broken pairs.

Let us define:

$$
n\left(l_{m n}\right)=\sum_{s, d} b_{m n}^{s d}
$$

$n\left(l_{m n}\right)$ is equal to the number of clear-channels having no disjoint alternate path in case of failure of the physical link $l_{m n}$. So $n\left(l_{m n}\right)$ represents the number of broken pairs that have $l_{m n}$ as a component.

A link $l_{m n}$ is considered to be critical if $n\left(l_{m n}\right) \geq 1$. A clear-channel $c_{s d}$ is protected if and only if any single link failure that causes $c_{s d}$ to fail leaves an alternate path to $c_{s d}$ not failed. A clear-channel $c_{s d}$ is protected iff $\sum_{m, n} b_{m n}^{s d}=0$ (from equation 5 ).

The function to minimize is then

$$
\mathcal{F}(R)=\sum_{m, n} n\left(l_{m n}\right)
$$

where

$$
R=\left(\mathcal{R}_{s d}\right)
$$

is the $N \times N$ network design matrix containing the routes $\mathcal{R}_{s d}$ of all the clearchannels, as shown in equation 3.

$\mathcal{F}(R)$ represents the number of broken pairs of the actual network design.

\section{The DAP Algorithm}

The effects of correlated failures of many clear-channels sharing each physical link can be eliminated - or at least minimized - by using the Disjoint Alternate Path (DAP) algorithm that we develop in this section. The DAP algorithm maps the clear-channels onto the physical network in such a way that, for each of the clear-channels (single-hop paths), there exists an alternate path (multi-hop) with same end-nodes, but sharing no optical link with its associated single-hop path.

The algorithm is explained in this section, and results of simulations on the ARPA2 network in section 4. These show the strong enhancement achieved in protection against the effects of optical link failures by the DAP algorithm compared to the one achieved by simple design strategies, such as a ShortestPath Routing algorithm.

The search for the best solution, the network mapping matrix $\mathrm{R}^{\star}$ which minimizes $\mathcal{F}$ (Equations 10 and 9), requires the evaluation of $\mathcal{F}$ for all the possible values of $R$. This means calculating, for each clear-channel, all the possible elementary routes between its two end-nodes in the physical network. Then the value of $\mathcal{F}$ must be computed for all the possible combinations of elementary routes for each clear-channel.

Let us define $X$ as the number of clear-channels in the virtual topology and $Y$ as the average number of possibilities to route a clear-channel in the physical topology. There are $Y^{X}$ possible configurations for which $\mathcal{F}$ has to be calculated 
in order to find its minimum value. As in real cases both $X$ and $Y$ grow nonlinearly with the number of nodes, the search of optimum solution for such problems soon becomes untractable. We have not tried to build an algorithm to find the optimum solution, but used a heuristic based on Tabu Search.

\subsection{The Realization}

The algorithm consists of routing the various clear-channels in a way such that none of them (if it is possible) or, at least, a minimum number of them are broken in case of any single optical link failure, while respecting the link capacity constraints. As explained earlier the Tabu Search heuristic is used by the DAP algorithm due to the very large number of possible solutions when the number of nodes in the network increases.

Tabu Search can be defined as a general heuristic in which a local search procedure is applied at each step of the general iterative process. It could be superimposed on other heuristics to prevent those being trapped in a local optimum. Tabu Search has been proposed at almost the same time by Glover [17] and Hansen [18] independently and, for interested readers, a recent paper [19] gives a detailed description of the method.

In our problem, Tabu Search starts from an initial mapping matrix $\mathcal{R}_{0}$ (given by randomly choosing routes on the physical network for all of the clearchannels) and explores the solution space in an iterative way by slightly modifying the route of a clear-channel to have a new mapping matrix. The main idea of Tabu Search is that, in order to avoid cycles trapping the algorithm in a local minimum, and also to enlarge the visited solution space, a Tabu List is created, which keeps track of the most recently visited solutions, i.e. the last changes performed on routes. Before moving to a new solution, the algorithm scans this list, and does not make the move if the new mapping is already in the list. So, a 'path' out of a local minimum can be found. The use of such a list also helps to diversify and intensify the search in the medium and long term.

Two features added to "plain" Tabu Search enlarge the visited solution space and reduce the number of iterations required to reach an optimum solution [20]. First, a randomly variable Tabu List size allows to alternate research intensification in a region (small size) to diversification (long size forbidding a lot of already tried moves). Secondly, the "aspiration criteria" allows to accept a move even if it is already present in the Tabu List, provided this move leads to a state whose value of $\mathcal{F}$ is smaller than the best solution found so far. This guarantees that the move, even if normally forbidden, leads to a state never visited before. 
The simplified DAP algorithm structure is the following one:

(1) Starts from an arbitrary solution.

(2) Randomly modifies the route of each clear-channel, with higher probability to avoid critical links.

(4) Keeps the solution which has the minimal F.

(5) Computes the new list of critical links based on the new matrix $R$.

(6) Starts the next step in (2).

\subsection{The Parameters}

The DAP algorithm uses the Tabu Search parameters as defined below:

Move A move $m_{s d}$ consists of rerouting the clear-channel $c_{s d}$ without using the links present in the movelist $m l_{s d}$.

Movelist A movelist $m l_{s d}$ is associated to each clear-channel $c_{s d}$. It consists of the list of all the links that have already been forbidden by a previous move, plus the new forbidden link, associated with this move. While no route for $c_{s d}$ can be found on the physical topology due to this constraint (a cutset [21] for $s$ and $d$ is included in $m l_{s d}$ ), the oldest included link of $m l_{s d}$ is removed. The reason for forbidding the use of a list of links instead of just a link is to enlarge the visited solution space by avoiding oscillation between two routes. The link to be added to the list is chosen randomly between all the links that are part of the actual route of this clear-channel, with a higher probability to choose a link $l_{i j}$ which causes $b_{i j}^{s d}=1$ (if there is one). The fact of choosing this link randomly renders cycling less probable and the higher probability of choosing a critical link directs the search faster towards good solutions.

Tabu List The tabu list is the list of $c_{s d}$ that have been chosen as best move for the last $t_{-}$size iterations.

Tabu List Size The tabu list size, $t_{-}$size, is varying between $\lfloor 0.9 \cdot \mathrm{mts}\rfloor$ and $\lceil 1.1 \cdot m t s+4\rceil^{1}[20]$, where $m t s$ is defined as a function of the number of clear-

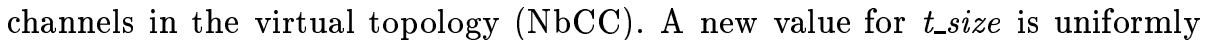
randomly chosen between the two bounds after every $2 \cdot\lceil 1.1 \cdot \mathrm{mts}+4\rceil$ iterations.

$m t s$ is set to $\mathrm{NbCC} / 4$. This value has been found by empirical testing, but the results with various $m t s$ values are not shown here, as the main aim of this paper is the description of Design Protection and a way to achieve it, and not the fine tuning of parameters.

\footnotetext{
${ }^{1}$ small problems need proportionally a longer tabu list than large ones. This is why there is a " $+4 "$ in the upper bound value.
} 
Stopping Criteria The stopping criteria maxitnb, defined as the maximum number of iterations allowed with no decrease of $\mathcal{F}$ over the best found solution until now, is also needed, as we do not know the theoretical minimum of $\mathcal{F}_{\text {calc }}$ (Equation 11 in the next paragraph).

maxitnb is set to $3 \cdot \mathrm{NbCC}$, but if a solution with $\mathcal{F}=0$ is found the algorithm of course stops.

Capacity Constraints The DAP algorithm tries to find a solution to the design problem which respects the capacity constraints. It does this in the following way: Violating capacity constraints is allowed, but it increases a lot the function to minimize. Strong enforcement of the capacity constraints has not been implemented, as it may confine the algorithm to a small "island" in the possible solution space, without any possibility to leave it by relaxing the capacity constraints. It also renders difficult the computation of the initial solution or the use of the algorithm if the capacity constraints cannot be respected by any solution. The DAP algorithm aims at minimizing the following function:

$$
\mathcal{F}_{\text {calc }}=\mathcal{F}+\mathcal{F}_{\mathcal{C}}
$$

where $\mathcal{F}$ is the one of equation 9 and $\mathcal{F}_{\mathcal{C}}$ is the addition due to capacity constraints violation, with

$$
\mathcal{F}_{\mathcal{C}}=\sum_{m, n} \max \left[0, \sum_{s, d}\left(r_{m n}^{s d}-l_{m n}\right)^{2}\right]
$$

\section{Results}

Two simples design algorithms, SPR-P and SPR-CC, are compared to DAP. By studying the results of the three algorithms on various topologies, we show that Design Protection is an important issue that should be addressed when mapping clear-channels in a WDM network, and that the DAP algorithm solves the problem. The three algorithms are:

DAP The Disjoint Alternate Path algorithm, as described in section 3.

SPR-CC The Shortest-Path Routing - Capacity Constraint algorithm. The use of SPR to perform the network design means that each clear-channel is placed on the physical topology using the shortest route [22], without any optimization towards resilience against link failures. Capacity constraints are, when possible, respected by SPR-CC. It always chooses the shortest route which minimizes the number of times the capacity constraints are violated. SPR-CC maps all the clear-channels on the physical network one after the other, the next one being randomly chosen among those not yet placed.

SPR-P The Shortest-Path Routing algorithm. The Shortest Path on the physical topology is chosen for each clear-channel. The Capacity Constraints 
are not taken into account in this algorithm, i.e. the algorithm acts as if infinite capacity was associated with all of the links.

\subsection{Experimental Results}

The physical topology used for the tests was the ARPA2 network, as shown in Figure 3. The cost associated with the use of a link is set to 1 for all the links, the length of a path corresponding in this case to the number of links it uses. The maximal number of wavelength channels per optical link is set to the same value $\Lambda_{\max }$ for all the existing links. It should be noted that the algorithm may also be used for problems where different values are associated with each optical link for the cost (like delays, distance between nodes) or the capacity (various types of fibers, or nodes with different number of transceivers).

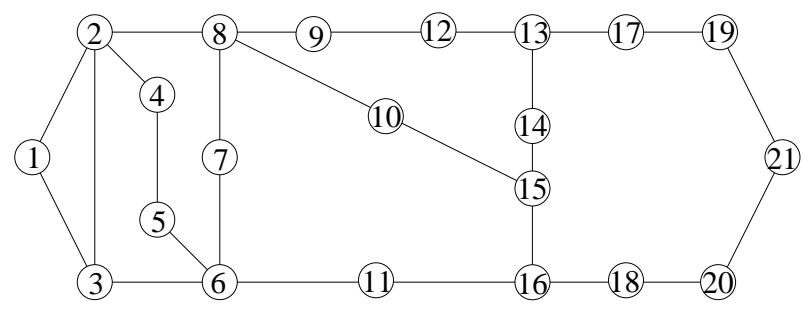

Figure 3: The 21-nodes 26-links ARPA2 physical network

The virtual topology has been defined by randomly generating clear-channels to obtain (at least) biconnected networks with various nodal degrees ${ }^{2}$.

SPR-P, SPR-CC and DAP have been implemented in C++ on a Sparc 20 workstation and applied to various virtual topologies on the ARPA2 physical network to be compared.

The values of $\mathcal{F}$ and $\mathcal{C}$ for the designs they propose are shown for various nodal degrees in Tables 1 to $3 . \mathcal{F}$ represents the number of broken pairs (cf. Equation 5) of the proposed design, $\mathcal{C}$ the number of times the capacity constraints are not respected, i.e. $\mathcal{C}=\sum_{m, n} \max \left[0, \sum_{s, d}\left(r_{m n}^{s d}-l_{m n}\right)\right]$ and $m u l$ is the number of clear-channels using the most $u$ sed link.

\subsection{Discussion}

Tables 1 to 3 show that SPR-P and SPR-CC do not protect the network against failure propagation. The DAP algorithm resolves the problem, as it finds a design with no failure propagation $(\mathcal{F}=0)$ in almost every case, whereas SPR$\mathrm{P}$ and even SPR-CC often get high values of $\mathcal{F}$. This can be seen in Table 1 for example where, for the virtual topology number 3 and with a $\Lambda_{\max }=6$, $\mathcal{F}_{D A P}=0$ whereas the best found value for SPR-CC and SPR-P is $\mathcal{F}=17$.

\footnotetext{
${ }^{2}$ The nodal degree of a node is the number of clear-channels having one end-point in it. The nodal degree of a network is the mean of its nodes' nodal degree
} 


\begin{tabular}{||c||c|c||c|c||c|c||c|c||}
\hline V. top. & \multicolumn{2}{|c||}{1} & \multicolumn{2}{|c|}{2} & \multicolumn{2}{|c|}{3} & \multicolumn{2}{|c||}{4} \\
\hline$\Lambda_{\max }$ & 6 & 7 & 6 & 7 & 6 & 7 & 6 & 7 \\
\hline $\mathcal{F}_{D A P}$ & $0-10$ & $0-2$ & $0-8$ & $0-2$ & 0 & $0-2$ & $2-9$ & $2-4$ \\
\hline $\mathcal{C}_{D A P}$ & $7-9$ & $0-1$ & $3-4$ & 0 & 0 & 0 & 0 & 0 \\
\hline$m u l_{D A P}$ & $7-8$ & $7-8$ & 7 & 7 & 6 & $6-7$ & 6 & 7 \\
\hline \hline $\mathcal{F}_{S P R-C C}$ & $11-40$ & $6-32$ & $9-35$ & $9-25$ & $17-23$ & $17-23$ & $20-34$ & $18-34$ \\
\hline $\mathcal{C}_{S P R-C C}$ & $7-14$ & $1-4$ & $4-6$ & $0-2$ & $0-1$ & 0 & $2-6$ & $0-1$ \\
\hline$m u l_{S P R-C C}$ & 8 & 8 & $7-8$ & $7-8$ & $6-7$ & 7 & $7-8$ & $7-8$ \\
\hline \hline $\mathcal{F}_{S P R-P}$ & 21 & 21 & 29 & 29 & 17 & 17 & 39 & 39 \\
\hline $\mathcal{C}_{S P R-P}$ & 15 & 8 & 11 & 8 & 4 & 2 & 10 & 5 \\
\hline$m u l_{S P R-P}$ & 10 & 10 & 12 & 12 & 8 & 8 & 9 & 9 \\
\hline \hline
\end{tabular}

Table 1: Results for a nodal degree of 3 for the virtual topology (32 clear-channels) on ARPA2 physical network: This table shows, for 4 different randomly generated virtual topologies and various values of $\Lambda_{\max }$, the minimal and maximal values found by the algorithms for the number of broken pairs $\mathcal{F}$, the number of times the capacity constraints have been violated by the solution $\mathcal{C}$, and the number of clear-channels using the most used link mul.

DAP finds solutions which are not only resilient against single link failures $(\mathcal{F}=0)$ but also respect capacity constraints better than those of SPR-P or even SPR-CC. This can be seen in table 3 where, for the virtual topology number 1 and with a $\Lambda_{\max }=10, \mathcal{C}_{D A P}=0$, whereas the best found solution of SPR-CC has $\mathcal{C}_{S P R-C C}=1$.

Note that if $\mathcal{F}_{D A P}$ or $\mathcal{C}_{D A P} \neq 0$, it does not mean that there is no solution, but that our heuristic has not found one. As a search through the whole solution space has not been perfomed, it is impossible to be sure that no solution exists.

It should be noted that as the nodal degree increases, solutions found by SPR-P and SPR-CC for $\mathcal{F}$ become better. This is due to the fact that the number of different multihop paths also increases with the number of clearchannels, and so the probability of finding one of them being disjoint with the singlehop path it is associated with is bigger. A larger nodal degree also speeds up the DAP algorithm, which finds a solution with $\mathcal{F}=0$ faster, as constraints on disjoint alternate paths are easier to fulfill.

\section{Conclusion}

WDM optical networks allow an optimal use of the fibre capacity by offering a wide bandwidth, necessary to respond to the needs of actual applications. However we are also witnessing a rapidly increasing concern about the reliability of the communications, as the consequences of a failure in high-speed networks are proportional to the amount of information flowing through the network.

In this article, we have argued that protection inside WDM networks can be performed at two levels: Physical and Design. 


\begin{tabular}{||c||c|c||c|c||c|c||c|c||}
\hline V. top. & \multicolumn{2}{|c||}{1} & \multicolumn{2}{|c|}{2} & \multicolumn{2}{|c|}{3} & \multicolumn{2}{|c||}{4} \\
\hline$\Lambda_{\max }$ & 7 & 8 & 7 & 8 & 7 & 8 & 7 & 8 \\
\hline \hline $\mathcal{F}_{D A P}$ & $0-8$ & 0 & 0 & 0 & 0 & 0 & 0 & 0 \\
\hline $\mathcal{C}_{D A P}$ & $8-9$ & $3-4$ & $3-4$ & 1 & $5-6$ & 0 & $6-7$ & 1 \\
\hline $\operatorname{mul}_{D A P}$ & 10 & $9-10$ & 9 & 9 & $8-9$ & 8 & 9 & 9 \\
\hline \hline $\mathcal{F}_{S P R-C C}$ & $3-28$ & $7-19$ & $8-21$ & $14-21$ & $0-21$ & $0-23$ & $13-22$ & $13-25$ \\
\hline $\mathcal{C}_{S P R-C C}$ & $8-16$ & $4-9$ & $3-5$ & 1 & $6-16$ & $0-4$ & $6-13$ & $1-4$ \\
\hline $\operatorname{mul}_{S P R-C C}$ & $9-12$ & $9-11$ & 9 & 9 & $8-9$ & $9-10$ & 9 & $9-10$ \\
\hline \hline $\mathcal{F}_{S P R-P}$ & 13 & 13 & 21 & 21 & 20 & 20 & 22 & 22 \\
\hline $\mathcal{C}_{S P R-P}$ & 15 & 8 & 5 & 2 & 16 & 10 & 12 & 6 \\
\hline $\operatorname{mul}_{S P R-P}$ & 11 & 11 & 10 & 10 & 11 & 11 & 11 & 11 \\
\hline \hline
\end{tabular}

Table 2: Results for a nodal degree of 4 for the virtual topology (42 clear-channels) on ARPA2 physical network: This table shows, for 4 different randomly generated virtual topologies and various values of $\Lambda_{\max }$, the minimal and maximal values found by the algorithms for the number of broken pairs $\mathcal{F}$, the number of times the capacity constraints have been violated by the solution $\mathcal{C}$, and the number of clear-channels using the most used link mul.

Physical Protection provides a reliable transport capability to the higher level networks. It is expensive to implement due to the need to duplicate all the optical hardware, but it extremely simple to control. For Physical Protection, it is the photonic network that is responsible for performing the necessary reconfiguration following a failure, and will do so on a very fast timescale.

Design Protection is a new kind of protection well adapted to WDM networks, where many channels share the same fibre. Design Protection does not guarantee carrying all the traffic that was carried prior to the component of optical link failure. Instead, it aims at maintaining connectivity between all pairs of network ports following a single failure, and lets the higher level network reconfigure itself so as to carry only the high priority traffic from the failed virtual channels. The fraction of the disrupted traffic that can be accommodated on the Alternate Path will be dependent on the loading of the higher level network.

The independence between the virtual and physical topologies offers a lot of possibilities to design clear-channels. We have shown the problems that failure propagation causes if the network design is realized without taking into account the interaction between the two levels. As the resolution of this problem is rather complex, a heuristic, the DAP algorithm, has been developed to find a good solution to this problem rapidly.

Experimental results have shown that by using this DAP algorithm to place the clear-channels at the design time, the network is much more resilient to single optical link failures than when clear-channels are placed using design algorithms which do not consider the problem, like SPR-P or SPR-CC for example.

Lastly, it should be noted that Design Protection applies to other settings like (1) an SDH network where the "physical topology" is made of fibres or clear-channels and the "virtual topology" is made of SDH end-to-end paths, 


\begin{tabular}{||c||c|c|c|c||c|c||c|c||}
\hline V. top. & \multicolumn{2}{|c||}{1} & \multicolumn{2}{c|}{2} & \multicolumn{2}{|c|}{3} & \multicolumn{2}{|c|}{4} \\
\hline$\Lambda_{\text {max }}$ & 9 & 10 & 9 & 10 & 9 & 10 & 9 & 10 \\
\hline \hline $\mathcal{F}_{D A P}$ & 0 & 0 & 0 & 0 & 0 & 0 & 0 & 0 \\
\hline $\mathcal{C}_{D A P}$ & $2-4$ & 0 & $12-14$ & 6 & $15-17$ & $8-7$ & 5 & 0 \\
\hline mul $_{D A P}$ & 10 & 10 & $12-13$ & 12 & $12-13$ & 12 & $10-11$ & 10 \\
\hline \hline $\mathcal{F}_{S P R-C C}$ & $0-21$ & $11-21$ & $0-30$ & $0-16$ & $0-13$ & $0-13$ & $11-19$ & $8-19$ \\
\hline $\mathcal{C}_{S P R-C C}$ & $6-10$ & $1-4$ & $17-27$ & $6-10$ & $16-22$ & $9-12$ & $5-8$ & $0-3$ \\
\hline $\operatorname{mul}_{S P R-C C}$ & $10-12$ & $11-12$ & $12-13$ & $12-13$ & $12-13$ & 13 & $10-11$ & $10-11$ \\
\hline \hline $\mathcal{F}_{S P R-P}$ & 24 & 24 & 16 & 16 & 13 & 13 & 17 & 17 \\
\hline $\mathcal{C}_{S P R-P}$ & 17 & 13 & 23 & 15 & 22 & 15 & 9 & 6 \\
\hline $\operatorname{mul}_{S P R-P}$ & 15 & 15 & 14 & 14 & 14 & 14 & 13 & 13 \\
\hline \hline
\end{tabular}

Table 3: Results for a nodal degree of 5 for the virtual topology (53 clear-channels) on ARPA2 physical network: This table shows, for 4 different randomly generated virtual topologies and various values of $\Lambda_{\max }$, the minimal and maximal values found by the algorithms for the number of broken pairs $\mathcal{F}$, the number of times the capacity constraints have been violated by the solution $\mathcal{C}$, and the number of clear-channels using the most used link mul.

(2) an ATM network where the "physical topology" is made of SDH paths and the "virtual topology" is made of ATM Virtual Paths connections or (3) an IP network, where the "physical topology" is made of ATM connections and the

"virtual topology" is made of adjacencies between routers.

\section{References}

[1] C. A. Brackett, "Dense Wavelength Division Multiplexing networks: Principles and Applications," Journal of Selected Areas in Communication, vol. 8, pp. 948-963, Aug. 1990.

[2] P. E. Green, "The Future of Fiber-Optic Computer Networks," IEEE Computer, Sept. 1991.

[3] G. Hill et al., "A transport network layer based on optical network elements," Journal of Lightwave Technology, vol. 11, pp. 667-679, May 1993.

[4] H. S. Hinton, "Photonic Switching Fabrics," IEEE Communications Magazine, pp. 71-89, Apr. 1990.

[5] R. Ramaswami, "Multiwavelength Lightwave Network for Computer Communication," IEEE Communications Magazine, pp. 78-88, Feb. 1993.

[6] B. Gavish and I. Neuman, "Routing in a Network with Unreliable Components," IEEE Transactions on Communications, vol. 40, pp. 1248-1257, July 1992 . 
[7] W. D. Grover, "Case Studies of Survivable Ring, Mesh and Mesh-Arc Hybrid Networks," in Globecom'92, vol. 1, pp. 633-638, Dec. 1992.

[8] T.-H. Wu, Fiber Network Service Survivability. Artech House, 1992.

[9] B. Mukherjee et al., "Some principles for designing a wide-area optical network," IEEE/ACM Transactions on Networking, vol. 4, pp. 684-696, Oct. 1996.

[10] R. Ramaswami and K. N. Sivarajan, "Desing of Logical Topologies for Wavelength-Routed Optical Networks," IEEE Journal on Selected Areas in Communications, vol. 14, pp. 840-851, June 1996.

[11] "Physical and networking layer issues of COBNET," Deliverable AC069/COB/WP1/DS/R/111/b1, COBNET Consortium, Mar. 1996.

[12] B. Mukherjee, "WDM-based local lightwave networks - part I: Multihop systems," IEEE Networks, pp. 12-27, May 1992.

[13] B. Mukherjee, "WDM-based local lightwave networks - part II: Multihop systems," IEEE Networks, pp. 20-32, July 1992.

[14] J. Armitage, O. Crochat, and J.-Y. Le Boudec, "Design of a survivable WDM photonic network," in Proc. of INFOCOM, Apr. 1997.

[15] M. Grotschel et al., Handbooks in OR and $M S$, vol. 7, ch. 10. Elsevier Science B. V., 1995.

[16] J.-F. P. Labourdette and A. S. Acampora, "Logically Rearrangeable Multihop Lightwave Networks," IEEE Transactions on Communications, vol. 39, pp. 1223-1230, Aug. 1991.

[17] F. Glover, "Future paths for integer programming and links to artificial intelligence," Computers and Operations Research, no. 13, pp. 533-549, 1986.

[18] P. Hansen, "The steepest ascent mildest descent heuristic for combinatorial programming," in Proc. Congr. on Numerical Method in Combinatorial Programming, Academic Press, 1986.

[19] F. Glover, E. Taillard, and D. de Werra, "A user's guide for tabu search," Annals of Operations Research, no. 41, pp. 3-28, 1993.

[20] E. D. Taillard, Recherches Iteratives Dirigees Paralleles. PhD thesis, EPFL / DMA, 1993. Thesis Nr 1153.

[21] B. Carre, Graphs and Networks. Oxford University Press, 1979.

[22] B. Mukherjee et al., "Some principles for designing a wide-area optical network," in Proc. of INFOCOM, vol. 1, pp. 110-119, 1994. 